\title{
Service evaluation in old age psychiatry: using the general practitioner's view
}

\author{
Eric GehlhaAR, Lecturer in Psychiatry, Academic Unit for of Old Age, St Charles \\ Hospital, London W10
}

Psychiatric services for the elderly are evolving rapidly. The Government has recently reiterated that this area needs special attention.' The reasons for upheaval are well-known: the changing age structure of the population, the historical neglect of the elderly mentally ill and a growing emphasis on communitybased approaches in place of traditional institutional solutions. In the London area and elsewhere, adjustment has been complicated by geographical transplantation from old suburban mental hospitals to locally situated units, often within district general hospitals. New specialist services also suffer from the effects of financial stringency and inadequate recruitment. These and other changes have ensured that the process of restructuring services has been difficult. Planners have had few precedents to fall back on, and have been heavily dependent on available guidelines. $^{2-4}$ There has been comparatively little information available about how effectively services work in practice. 5

The most popular model for a contemporary psychogeriatric service is ambitious. This "seeks to tackle all mental disorders presented for specialist attention from a defined population and to provide a comprehensive response to their needs". ${ }^{2}$ Arie \& Jolley have suggested five basic principles underpinning good practice: flexibility; responsiveness and availability; unhierarchical use of staff; domiciliary assessment; and willingness to collaborate with other services and agencies. These principles can be used as a basis for a simple form of service evalution. Two aspects need to be considered. Firstly, are these principles acceptable and worthwhile? Secondly, how far does any individual service which espouses them succeed in its objectives?

\section{The service}

Paddington and North Kensington District (PNK) has faced many of the above challenges. It is a small inner city district with a population of 125,000 . About $14 \%$ of the resident population are aged 65 or over (i.e. approximately 17,500 ), of whom $40 \%$ live alone. Previously, elderly patients from the district had been admitted to a large mental hospital on the south-western fringe of London; few services had been situated within the district. In 1985 , a new service was introduced, and a purpose built unit was opened on the site of a district general hospital. The new service aimed to provide a comprehensive range of assessment and treatment facilities (short-stay and long-stay wards, day hospital, out-patient clinic) for all psychiatric patients over 65 , irrespective of diagnosis.

\section{Referral procedure}

Since September 1985, the Community Assessment Team has seen all newly referred patients aged 65 or over. Patients are accepted from GPs other doctors, community nurses, social workers, home helps and other professionals. Self-referrals are not accepted. When the referrer is not the patient's GP, we discuss the referral with the GP first. Referrals are made by letter or telephone. There is a daily meeting where two team members are selected to carry out an initial assessment on the basis of availability and a provisional judgement about the patient's needs. Urgent referrals are accepted only from medical staff and can be seen on the day of referral at home or in the casualty department. There are two assessment teams which include four doctors (consultant, senior registrar, clinical assistant and registrar/SHO), a community psychiatric nurse, a social worker and occupational therapist. Each team member has other responsibilities and may devote two to five hours per week to new referrals. Medical students and other trainee professionals are attached to the team for training purposes. There is a weekly review meeting in which initial assessments are discussed and future plans made.

\section{Referral patterns}

The unit receives about 50 referrals per month. Just under half are in-patients in other hospital wards. These are typically seen one to four days after the allocation meeting. Other patients are seen almost invariably at home in the first instance, usually three to seven days after the allocation meeting. Occasionally patients are seen in the out-patient department. The present practice is that a doctor virtually always serves as an initial assessor. Surveys of referrals in 
autumn 1985, and spring 1987 have shown a consistent pattern of diagnosis: $40-50 \%$ have dementia, $30-35 \%$ have affective disorders (depression or mania), $20 \%$ have other psychiatric disorders (schizophrenia, confusional state, neurosis, personality disorder or alcohol abuse), and 5-10\% have no psychiatric disorder; $70 \%$ of patients seen are female.

\section{GPs as consumers}

A strong emphasis on service evaluation existed within the unit throughout its first two years of operation. This was reinforced by the development of a professorial academic unit from April 1986 and the appointment of another consultant as part-time subunit general manager. The difficulties of service evalution are well known. Performance indicators are published after a considerable delay and therefore cannot be used to evaluate a rapidly changing service. Many traditional measures are irrelevant or misleading in a service based firmly on domiciliary assessment. Moreover, the viewpoint of the "consumers' needs to be heard. Interest in this aspect of service evaluation is reflected at District level by the development of quality assurance research. There are many different 'consumers' of any service, both professional and non-professional. Among these consumers, it can be argued that GPs have a particularly useful and distinctive role. They are closely in touch with other elements of community care, and can be seen as advocates of patients and their relatives. Many are able to compare the present situation with the previous service. They are also able to compare management of different types of cases.

\section{Method}

I identified all GPs known to the Family Practitioners Committee or our department who attended patients in the PNK district, approximately 100 in all. A questionnaire was sent in April 1987 with a reminder the following month. The FPC was out of date, and some local practitioners offered services to a very restricted number or range of PNK residents. There were 38 replies received, of which 30 answered all questions. This response rate represented two thirds of those who referred cases with any regularity.

\section{Results}

Referrals procedure: This seemed to work well, with $95 \%$ of respondents finding the procedure convenient and easily understandable. Another aspect of accessibility in the service was its acceptance of referrals from non-medical professionals (although the recommended policy was for non- medical referrers to contact the GP involved for prior permission). Perhaps surprisingly, this procedure was acceptable to all respondents.

Domiciliary assessment: All GPs agreed with our emphasis on domiciliary assessment in virtually all patients not already in hospital; $26 \%$ had certain reservations, most centred around delays. One commented that home visits were sometimes unnecessary, while another felt that some patients would prefer out-patient clinics.

Responsiveness: Only $14 \%$ of GPs felt that referrals were delayed unnecessarily. However, analysis of comments suggested that a larger number found the service too slow. The most frequent complaint was that more patients should have been seen on the day of referral, usually because of the possible need for admission.

Liaison: Two questions were asked, concerning communication and responsibility. Fifty per cent of GPs had "sometimes" or "often" perceived problems over responsibility for the patient, for example for prescription or continued assessment. In some cases, there was misunderstanding over whether the psychogeriatric service was still involved. Forty-five per cent of GPs did not feel that they received communications rapidly or frequently enough. One mentioned two cases of patients being discharged without his knowing until a crisis had occurred. Another pointed out the importance of the initial report being sent promptly. Two GPs reported that communications on long-term cases, especially in the day hospital, were irregular and scanty. There was more general satisfaction with the content of assessment letters: $93 \%$ found them to be relevant.

Multidisciplinary assessment: over $90 \%$ of GPs had no reservations about specialist assessments being done by trainee psychiatrists. Those who did doubted that SHO/registrars had superior expertise to the GPs themselves. One offered a more pragmatic reason: he found junior psychiatrists less able or willing to arrange admission than consultants! In contrast, there was considerable suspicion of the use of non-medical disciplines (social worker, community psychiatric nurse, occupational therapist) in the initial home assessment. Analysis of comments suggested that most reservations would be reduced if doctors accompanied other disciplines or were readily available for secondary referral within the unit. But one GP, who shared health centre premises with community psychiatric nurses, commented very favourably on their service.

General: There was general satisfaction with the service: $73 \%$ rated it as good or very good, and $85 \%$ felt that psychiatric care of the elderly had improved since its introduction.

A number of GPs made specific comments including: problems in persuading patients to attend day hospital and day centres; possibility of medically ill 
'confused' patients falling between psychiatric and geriatric services; assessors tend to underestimate ill health; criteria for urgent admission should be acute failure of community to cope rather than our own 'abstract criteria'; and two specific complaints about cases. In one, the GP felt that we had left too much responsibility with the family in finding a private nursing home. In the other, the GP criticised our refusals to treat under compulsory order a demented patient who was putting her life at risk by refusing surgery.

\section{Comments}

This survey was designed to have direct relevance to the local service. A number of areas were revealed which merited review by our unit: the need for same day assessment, the usefulness of prompt communications, greater explicitness over boundaries and sharing of responsibility, and suspicion of the role of non-medical involvement in assessment. In this way, a survey of GPs proved a useful and cost-effective way of evaluating a new unit. The results needed careful interpretation. Global approval ratings might more accurately reflect politeness or personal relationships than real changes in the quality of service. Specific complaints may reflect genuine shortcomings or idiosyncratic expectations. In this study, a number of GPs appeared to regard the previous system with nostalgia, presumably because patients were more readily admitted, less often discharged, and therefore caused fewer problems. In interpreting the results, the consistency of each judgement must be borne in mind both in terms of the survey itself and in comparison with other formal and informal mechanisms of feedback.
The results have wider significance. Other districts with similar objectives may find the specific areas of difficulty of relevance. Moreover, the survey method may be useful to other groups. More broadly, some judgements about Arie \& Jolley's ${ }^{2}$ principles can be made. Most appear to be acceptable to GPs, although responsiveness and full, clear collaboration may be hard to achieve. One principle which may need more thought is the un-hiearchical use of staff in a multidisciplinary team. It is unclear whether suspicion of non-medical disciplines results from unfamiliarity or reflects real disadvantages from the GP's viewpoint. If the former, then it is a public relations problem but if the latter, many services will need to re-evaluate their systems.

\section{References}

${ }^{1}$ Government Response to the Second Report from the Social Services Committee, 1984-85 session: Community Care with Special Reference to the Adult Mentally Ill and Mentally Handicapped People (1985) (Cmnd. 9674). London: HMSO.

${ }^{2}$ ARIE, \& JOLLEY, D. (1982) Making services work: organisation and style of psychogeriatric services In The Psychiatry of Late Life, (eds, R. Levy \& F. Post) Oxford: Blackwell.

${ }^{3}$ HORROCKS, P. (1986) The components of a comprehensive district health service for elderly people - a personal view. Age and Ageing, 15, 321-342.

${ }^{4}$ National Health Service Health Advisory Service (1983) The Rising Tide. London: Department of Health \& Social Security.

${ }^{5}$ Wattis, J., WATtIS, L. \& ARIE, T. (1981) Psychogeriatrics: a national survey of a new branch of psychiatry. British Medical Journal, 282, 1529-1533.

\title{
Psychiatrists' use of investigations
}

\author{
Anthony White, Senior Registrar in Psychiatry, Glenside Hospital, Stapleton, Bristol
}

Many westernised countries have introduced Quality Assurance (QA) into their process of health care. The UK has lagged behind other countries and psychiatry has lagged behind other specialities.

In 1980 the Australian Department of Health formed a seven member QA project team run by the Royal Australian and New Zealand College of Psychiatrists in an attempt to establish a methodology for QA in psychiatry. Using three sources of information: literature review, survey of views of practising psychiatrists and the views of an expert committee, they have now reported on the main categories of psychiatric illness. Their more recent reports have enjoyed publication in this country, suggesting the findings are of interest and relevance to us. ${ }^{1}$

Absent from the Australia and New Zealand QA surveys is any appraisal of physical illness in the 